\title{
Feedback Sensitivity of Distributed-Feedback Laser Diodes in Terms of Longitudinal Field Integrals
}

\author{
Geert Morthier, Senior Member, IEEE
}

\begin{abstract}
Using an alternative rate-equation description, analytical expressions are derived for the change of threshold gain, oscillation frequency and linewidth due to weak external optical feedback in distributed feedback laser diodes.
\end{abstract}

Index Terms-External feedback, optical communication, semiconductor lasers.

\section{INTRODUCTION}

$\mathbf{T}$ HE INFLUENCE of external optical feedback on the behavior of laser diodes has been extensively studied since 1980, when Lang and Kobayashi [1] published the first experimental and theoretical analysis. The Lang-Kobayashi (LK) rate equations have, e.g., been used to derive expressions for the change of the threshold gain $\Delta G_{\mathrm{th}}$, of the oscillation frequency $\Delta \omega$, and of the laser linewidth $\Delta \nu$ due to weak external feedback

$$
\begin{aligned}
& \Delta G_{\mathrm{th}}= 2\left|C_{L, R}\right|\left|r_{\mathrm{ext}}\right| \cos \left(\omega \tau+\arg \left[C_{L, R}\right]\right) \\
& \Delta \omega \tau= \omega \tau-\omega_{0} \tau=-\left|C_{L, R}\right|\left|r_{\mathrm{ext}}\right| \tau \sqrt{1+\alpha^{2}} \\
& \cdot \sin \left(\omega \tau+\arg \left[C_{L, R}\right]+t g^{-1} \alpha\right) \\
& \Delta \nu=\Delta \nu_{0}\left[1+\left|C_{L, R}\right|\left|r_{\mathrm{ext}}\right| \tau \sqrt{1+\alpha^{2}}\right. \\
&\left.\cdot \sin \left(\omega \tau+\arg \left[C_{L, R}\right]+t g^{-1} \alpha\right)\right]^{-2}
\end{aligned}
$$

where $\tau$ is the round-trip time in the external cavity, $\alpha$ is the linewidth-enhancement factor, and $C_{L, R}$ is the feedback sensitivity for the facet on the left-hand and right-hand sides, respectively. $r_{\text {ext }}$ is the field reflection of the external reflector.

However, the LK equations are strictly valid only for FabryPerot laser diodes, in which case analytical (real) expressions are readily written down for $C_{L, R}$. In general, the relations (1) are assumed to be valid also for DFB lasers, whereby the feedback sensitivities $C_{L, R}$ are now defined by these relations [2]. $C_{L, R}$ are then usually calculated numerically by deriving $\Delta G_{t h}$ and $\Delta \omega$ from numerical laser simulations.

A more general and theoretical approach has been used by Nilsson and Buus [3], who showed that for all laser diodes

$$
\begin{aligned}
\left|C_{L}\right|+\left|C_{R}\right| & =\frac{2}{\tau_{\text {int }}} \alpha_{\text {end }} L \sqrt{K_{z}} \\
\frac{\left|C_{L}\right|}{\left|C_{R}\right|} & =\frac{\eta_{L}}{\eta_{R}}
\end{aligned}
$$

Manuscript received June 7, 2002. This work was supported by the DWTC under the IAP5/18 Project.

The author is with the Department of Information Technology, Ghent University-IMEC, B-9000 Gent, Belgium (e-mail: morthier@intec.rug.ac.be).

Publisher Item Identifier 10.1109/JQE.2002.802958

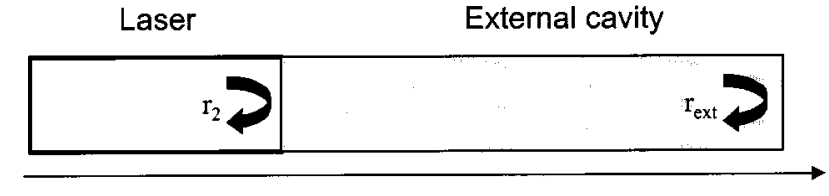

Fig. 1. Schematic structure of a laser diode with an external cavity $(\tau=$ $\left.L_{\mathrm{cxt}} / v_{g}\right)$.

where $\alpha_{\text {end }}$ is the mirror loss, $L$ is the diode length, and $K_{z}$ is the longitudinal Petermann factor [4]. $\eta_{L, R}$ are the extraction efficiencies for the left- and right-hand-side facets, and $\tau_{\text {int }}$ is the round-trip time in the laser cavity. Nilsson and Buus proved the relations (2) only for the influence of the feedback on the laser linewidth. Moreover, in the meantime, it has been shown that the linewidth depends on an effective linewidth enhancement factor $a_{\mathrm{eff}}$ and not on $\alpha$. To date, it has not been clarified whether one also has to use $a_{\text {eff }}$ to derive the influence of feedback on the change of the oscillation frequency or whether the values for $C_{L, R}$ given in (2) can be used in all of the relations (1).

In this paper, we derive exact expressions for $\Delta G_{\mathrm{th}}, \Delta \omega$, and $\Delta \nu$ based on an alternative rate-equation description for distributed feedback (DFB) lasers. The rate-equation description was published several years ago and has already been shown to lead to exact analytical expressions for the chirp and the harmonic distortion in DFB lasers [5]. Recently, we also used this rate-equation description to derive the influence of amplified spontaneous emission (ASE) on linewidth and relative intensity noise (RIN) of a laser diode integrated with a semiconductor optical amplifier (SOA) [6].

In Section II, we will derive the general, complex rate equation for a laser diode with weak external feedback. We take the facet facing the external feedback as the right-hand-side facet (at $z=L$ ). We then use this rate-equation description to derive exact expressions for $\Delta G_{\mathrm{th}}, \Delta \omega$, and $\Delta \nu$, and show that for a DFB laser, they can be expressed in terms of $K_{z}$ and the longitudinal confinement factor.

\section{RATE EQUATIONS FOR A LASER With EXTERNAL OPTICAL FEEDBACK}

The schematic structure of the laser diode with external feedback is shown in Fig. 1. To derive the alternative rate equations in the case of weak optical feedback, we use the approach outlined in [5]. The complex amplitudes $R^{+}(z, t)$ and $R^{-}(z, t)$ of 
the forward- and backward-propagating fields and the complex Bragg deviation $\Delta \beta(z, t)$ are expanded as

$$
\begin{aligned}
& R^{ \pm}(z, t)=R_{0}^{ \pm}(z)+\delta R^{ \pm}(z, t) \\
& \Delta \beta(z, t)=\Delta \beta_{0}+\delta(\Delta \beta(z, t))
\end{aligned}
$$

where $R_{0}$ and $\Delta \beta_{0}$ are the field amplitudes and Bragg deviation under static operation and in the absence of external feedback. The new rate equation can then be obtained after manipulation of the coupled wave equations (cf. [5]). Due to the external optical feedback (field reflection $r_{\text {ext }}$ ), one has to take into account modified boundary conditions at the right-hand-side facet $(z=L)$ as follows:

$$
\begin{aligned}
\delta R^{-}(L, t)=r_{2} e^{-2 j \beta_{0} L} & \delta R^{+}(L, t) \\
& +t_{2}^{2} r_{\text {ext }} R^{+}(L, t) e^{-2 j \beta_{0} L} e^{-j \omega \tau}
\end{aligned}
$$

where $\beta_{0}$ is the Bragg wavelength and $r_{2}$ and $t_{2}$ are the field reflection and transmission, respectively, for the right-hand-side facet. Assuming that the spatial profiles of $\delta R^{+}$and $\delta R^{-}$are identical to those of the static fields, we can further write

$$
\delta R^{ \pm}(z, t)=R_{0}^{ \pm}(z)\{\sqrt{1+f(t)}-1\}
$$

and

$$
\delta S(z, t)=S_{0}(z) f(t)
$$

Here, $S(z)$ is the photon density in the laser cavity. Combination of the time-dependent coupled-wave equations for $\delta R^{+}$ and $\delta R^{-}$with the static coupled-wave equations and integration from $z=0$ (left-hand-side laser facet) to $z=L_{d}$ (righthand-side laser facet) yields the following equation:

$$
\begin{aligned}
j \frac{\partial \sqrt{1+f}}{\partial t}+v_{g} F_{c}-j \frac{v_{g}\left[R_{0}^{+}(L)\right]^{2}}{2 \int_{0}^{L} d z R_{0}^{+} R_{0}^{-}} \\
\quad \cdot t_{2}^{2} r_{\mathrm{ext}} \sqrt{1+f} e^{-j \omega \tau} e^{-2 j \beta_{0} L} \\
=\sqrt{1+f}\left\{\Delta \omega+j \frac{1+j \alpha}{2} G^{\prime}\left[\delta N_{0}+\delta N_{1}(\chi-1)\right]\right\} .
\end{aligned}
$$

$G^{\prime}$ is the differential gain and $v_{g} F_{c}$ is a Langevin force representing spontaneous emission.

For the sake of simplicity, we have neglected the nonlinear gain, and the carrier density has been expanded as $N(z, t)=$ $N_{0}(t)+N_{1}(t)\left[\left(S_{0}(z)-S_{\mathrm{av}}\right) / S_{\mathrm{av}}\right]$, where $S_{\mathrm{av}}$ is the spatial average of $S_{0}$. The second-order moment of $v_{g} F_{c}$ is given by

$$
\left\langle v_{g} F_{c}(t) F_{c}^{*}\left(t^{\prime}\right) v_{g}\right\rangle=G n_{\mathrm{sp}} \frac{K_{z}}{S_{\mathrm{av}} V_{\mathrm{act}}} \delta\left(t-t^{\prime}\right)
$$

where $V_{\text {act }}$ is the active layer volume, $G$ is the modal gain (per $s$ ), and $n_{\mathrm{sp}}$ is the inversion factor of the laser. $\chi$ is a complex factor that describes the influence of spatial hole burning and is a function of the field distributions in the laser cavity only [5]

with

$$
\chi=\chi^{\prime}+j \chi^{\prime \prime}=\int_{0}^{L} d z \frac{S_{0}(z)}{S_{\mathrm{av}}} \Gamma_{z}(z)
$$

$$
\Gamma_{z}(z)=\frac{R_{0}^{+}(z) R_{0}^{-}(z)}{\int_{0}^{L} R_{0}^{+}(z) R_{0}^{-}(z) d z}
$$

where $\Gamma_{z}(z)$ is the longitudinal confinement factor.

The rate equation (6) has to be combined with the carrier density rate equations for the derivation of laser characteristics. We will not provide the derivation of the rate equations for $N_{0}$ and $N_{1}$ here and refer to [5].

\section{Solutions of RATE EQuATION (5)}

To simplify the derivation further, we further assume that $f$ is small (weak optical feedback, weak noise source) and linearize (6). We also consider only the low-frequency dynamics and take the real and imaginary part of (6) as follows:

$$
\begin{aligned}
\Delta \omega= & \frac{\alpha}{2} G\left[\delta N_{0}+\delta N_{1}\left(\chi^{\prime}-1\right)\right]+\frac{G^{\prime}}{2} \delta N_{1} \chi^{\prime \prime} \\
& \quad+\operatorname{Re}\left\{v_{g} F_{c}\right\}+\frac{v_{g}\left|t_{2}^{2} r_{\mathrm{ext}}\left[R_{0}^{+}(L)\right]^{2}\right|}{\left|2 \int_{0}^{L} d z R_{0}^{+} R_{0}^{-}\right|} \sin \left(\omega \tau+\phi_{c}\right) \\
\frac{G^{\prime}}{2}\left[\delta N_{0}+\delta N_{1}\left(\chi^{\prime}-1-\alpha \chi^{\prime \prime}\right)\right] & \\
= & \operatorname{Im}\left\{v_{g} F_{c}\right\}-\frac{v_{g}\left|t_{2}^{2} r_{\mathrm{ext}}\left[R_{0}^{+}(L)\right]^{2}\right|}{\left|2 \int_{0}^{L} d z R_{0}^{+} R_{0}^{-}\right|} \cos \left(\omega \tau+\phi_{c}\right) \\
\phi_{c}= & \arg \left[\frac{t_{2}^{2} r_{\mathrm{ext}}\left[R_{0}^{+}(L)\right]^{2}}{2 \int_{0}^{L} d z R_{0}^{+} R_{0}^{-}}\right]-2 \beta_{0} L, \quad \frac{\left|t_{2}^{2}\right|\left|R_{0}^{+}(L)\right|^{2}}{\left|2 \int_{0}^{L} d z R_{0}^{+} R_{0}^{-}\right|} \\
= & \frac{\left|t_{2}^{2}\right|\left|R_{0}^{+}(L)\right|^{2}}{\int_{0}^{L} d z\left[\left|R_{0}^{+}\right|^{2}+\left|R_{0}^{-}\right|^{2}\right]} \frac{\int_{0}^{L} d z\left[\left|R_{0}^{+}\right|^{2}+\left|R_{0}^{-}\right|^{2}\right]}{\left|2 \int_{0}^{L} d z R_{0}^{+} R_{0}^{-}\right|} \\
= & \frac{P_{\mathrm{out}, r}}{P_{\mathrm{av}} L} \sqrt{K_{z \cdot}}
\end{aligned}
$$

It can be shown [5] that, in the low-frequency regime, $\delta N_{0}=\delta N_{1}$ such that the change in the (longitudinally averaged) threshold gain $\Delta G_{\mathrm{th}}=G^{\prime} \delta N_{0}$ is found to be

$$
\Delta G_{\mathrm{th}}=-\frac{2 v_{g}\left|r_{\mathrm{ext}}\right|}{\left(\chi^{\prime}-\alpha \chi^{\prime \prime}\right)} \frac{P_{\mathrm{out}, r}}{P_{\mathrm{av}} L} \sqrt{K_{z}} \cos \left(\omega \tau+\phi_{c}\right)
$$

while substitution of the values of $\delta N_{0}=\delta N_{1}$ in the equation for $\Delta \omega$ gives

$$
\begin{aligned}
\Delta \omega= & \frac{v_{g}\left|t_{2}^{2} r_{\mathrm{ext}}\left[R_{0}^{+}(L)\right]^{2}\right|}{\left|2 \int_{0}^{L} d z R_{0}^{+} R_{0}^{-}\right|} \\
& \cdot\left\{\sin \left(\omega \tau+\phi_{c}\right)-\frac{\alpha \chi^{\prime}+\chi^{\prime \prime}}{\chi^{\prime}-\alpha \chi^{\prime \prime}} \cos \left(\omega \tau+\phi_{c}\right)\right\} \\
= & v_{g}\left|r_{\mathrm{eff}}\right| \frac{P_{\mathrm{out}, r}}{P_{\mathrm{av}} L} \sqrt{K_{z}} \sqrt{1+\alpha_{\mathrm{eff}}^{2}} \\
& \cdot \sin \left(\omega \tau+\phi_{c}+t g^{-1}\left(\alpha_{\mathrm{eff}}\right)\right) \\
= & \frac{X}{\tau} \sin \left(\omega \tau+\phi_{c}+t g^{-1}\left(\alpha_{\mathrm{eff}}\right)\right) .
\end{aligned}
$$

The last equality defines $X$. Finally, for the linewidth one finds after some calculations

$$
\begin{aligned}
\Delta \nu=\frac{R_{\mathrm{sp}} K_{z}\left(1+\alpha_{\mathrm{eff}}^{2}\right)}{4 \pi S_{\mathrm{av}} V_{\mathrm{act}}} & \frac{1}{\left[1+X \sin \left(\omega \tau+\phi_{c}+t g^{-1}\left(\alpha_{\mathrm{eff}}\right)\right)\right]^{2}} .
\end{aligned}
$$


From (10)-(12), one can conclude that similar, but nevertheless slightly different equations such as (1), are still valid for DFB lasers. That is

$$
\begin{aligned}
\Delta G_{\mathrm{th}}= & 2 \frac{\left|C_{L, R}\right|}{\chi^{\prime}-\alpha \chi^{\prime \prime}} \cos \left(\omega \tau+\phi_{c}\right) \\
\Delta \omega \tau= & \omega \tau-\omega_{0} \tau=-\left|C_{L, R}\right| \tau \sqrt{1+\alpha_{\mathrm{eff}}^{2}} \\
& \cdot \sin \left(\omega \tau+\phi_{c}+t g^{-1}\left(\alpha_{\mathrm{eff}}\right)\right) \\
\Delta \nu=\Delta \nu_{0}\left[1+\left|C_{L, R}\right| \tau \sqrt{1+\alpha_{\mathrm{eff}}^{2}}\right. & \left.\cdot \sin \left(\omega \tau+\phi_{c}+t g^{-1}\left(\alpha_{\mathrm{eff}}\right)\right)\right]^{-2}
\end{aligned}
$$

with

$$
\left|C_{L, R}\right|=v_{g}\left|r_{\mathrm{ext}}\right| \frac{P_{\mathrm{out}, l, r}}{P_{\mathrm{av}} L} \sqrt{K_{z}}
$$

and

$$
\left|C_{L}\right|+\left|C_{R}\right|=\frac{2}{\tau_{\text {int }}} \alpha_{\text {end }} L \sqrt{K_{z}} .
$$

That is, we have proven the relations (2), derived earlier by Nilsson and Buus, in another (and more exact) manner. Moreover, we have shown that one has to use the effective linewidthenhancement factor $\alpha_{\text {eff }}$ [7] in the formulas for the linewidth and emission frequency change. Finally, the exact expression for the change of the threshold gain appears to be slightly different than what has so far been used.

Calculations of the effective linewidth enhancement factor, the longitudinal Petermann factor and of $\chi$ for different types of DFB laser diodes have been published before [4]-[7]. All of these parameters can be calculated with simple DFB laser models. It can easily be seen that structures which minimize the linewidth (through the effective linewidth-enhancement factor) will also minimize the sensitivity to external optical feedback.

\section{CONCLUSION}

We have given an exact derivation for the changes in threshold gain, emission wavelength, and linewidth of a DFB laser due to the presence of weak external optical feedback. It has been shown that the feedback sensitivity of DFB lasers can be expressed in terms of longitudinal field averages, such as those needed to calculate the effective linewidth-enhancement factor.

Although we have only addressed the case of weak external feedback, it can be expected from the derivation that the above defined feedback sensitivity will also determine the behavior of DFB lasers under stronger feedback, and particularly feedback from reflectors at longer distances. Our derived complex field rate equation (6) can furthermore also be used to investigate the influence of feedback on the RIN.

\section{REFERENCES}

[1] R. Lang and K. Kobayashi, "External optical feedback effects on semiconductor injection laser properties," IEEE J. Quantum Electron., vol. QE-16, pp. 347-355, 1980.

[2] F. Favre, "Theoretical analysis of external optical feedback on DFB semiconductor lasers," IEEE J. Quantum Electron., vol. QE-23, pp. 81-88, Jan. 1987.

[3] O. Nilsson and J. Buus, "Linewidth and feedback sensitivity of semiconductor diode lasers," IEEE J. Quantum Electron., vol. 26, pp. 2039-2042, Dec. 1990.

[4] J. Wang, N. Schunk, and K. Petermann, "Linewidth enhancement for DFB lasers due to longitudinal field dependence in the laser cavity," Electron. Lett., vol. 23, pp. 715-716, 1987.

[5] G. Morthier, "An accurate rate-equation description for DFB lasers and some interesting solutions," IEEE J. Quantum Electron., vol. 33, pp. 231-237, Feb. 1997.

[6] G. Morthier and B. Moeyersoon, "Intensity noise and linewidth of laser diodes with integrated semiconductor optical amplifier," IEEE J. Quantum Electron., to be published.

[7] M.-C. Amann, "Linewidth enhancement in distributed feedback semiconductor lasers," Electron. Lett., vol. 26, pp. 569-571, 1990.

Geert Morthier (SM'93) received the M.S. degree in electrical engineering and the Ph.D. degree from Ghent University, Gent, Belgium, in 1987 and 1991, respectively.

Since 1991, he has been a member of the permanent staff of the Interuniversity MicroElectronic Center (IMEC), Ghent University. During 1998-1999, he was the Project Manager of the EU ACTS project ACTUAL, dealing with the control and applications of widely tunable laser diodes. His main research interests are in the modeling and characterization of optoelectronic components, and he has authored or coauthored over 70 papers in the field. He is the coauthor of Handbook of Distributed Feedback Laser Diodes (Norwood, MA: Artech House: 1997) and coeditor of the book How to Model and Measure Photonic Components: Experience From a European Project (New York: Springer-Verlag, 1998). 